\title{
THE BERGMANN-SHILOV BOUNDARY OF A BOUNDED SYMMETRIC DOMAIN
}

\author{
M. MACKEY AND P. MELLON \\ UNIVERSITY COLLEGE DUBLIN
}

\begin{abstract}
We show that there are many sets in the boundary of a bounded symmetric domain that determine the values and norm of holomorphic functions on the domain having continuous extensions to the boundary. We provide an analogue of the Bergmann-Shilov boundary for finite rank $J B^{*}$-triples.
\end{abstract}

\section{INTRODUCTION}

Recall that an open unit ball $B$ of a complex Banach space $Z$ is homogeneous with respect to biholomorphic mappings if, and only if, $Z$ carries an algebraic structure that renders it a $J B^{*}$-triple, defined below [8]. $J B^{*}$-triples include Hilbert spaces, $C^{*}$-algebras and the classical Hermitian symmetric spaces known as Cartan factors. Bounded symmetric domains are the infinite dimensional analogues of the Hermitian symmetric spaces but, by Kaup's Riemann Mapping Theorem [8], we may alternatively introduce them as those domains in a Banach space which are biholomorphically equivalent to the unit ball of a $J B^{*}$-triple. This then is a natural category of Banach spaces in which to study holomorphic functions and demonstrates a rich interplay of complex and functional analysis, geometry and non-associative algebraic structures.

The first section of this paper consititutes a very brief introduction to the basic facts about $J B^{*}$-triples, including the concrete description of the biholomorphic mappings (automorphisms) of the unit ball in terms of the triple product. Tripotents and their order structure are introduced and linked to the extreme points of the unit ball. In Section 2 we prove that the automorphisms (which naturally extend to the boundary) preserve the set of extreme points as well as the unitary tripotents.

These facts allow us in Section 3 to express the closed unit ball as the closed convex hull of the set of maximal and unitary tripotents,

Date: December 15, 2021. 
when these sets are non-empty and, more generally, as the closed convex hull of the orbit of any $v \in \partial B$ under Aut $(B)$, denoted $G_{v}$, or its orbit under the connected identity component of Aut $(B)^{0}$, denoted $G_{v}^{0}$.

In Section 4 we see that any holomorphic function on $B$ which extends continuously to the boundary has its values inside the ball, and hence its norm, determined by its values on certain subsets of the boundary, which we call determining sets. We show that such determining sets include the set of extreme points and the set of unitary tripotents (if these exist) and, more generally, the orbits $G_{v}$ and $G_{v}^{0}$, of any $v \in \partial B$. We recall the notion and structure of boundary components of a finite rank $J B^{*}$-triple. We prove that automorphisms of $B$ map holomorphic boundary components onto holomorphic boundary components and, more importantly, they preserve the rank of such boundary components.

Section 5 culminates in our main result, namely, that the set of extreme points of a finite rank triple acts analagously to the BergmannShilov boundary in finite dimensions by providing the smallest closed subset of $\bar{B}$ which determines the norm of all holomorphic functions of the ball which have a continuous extension to the boundary.

\section{NOTATION AND BACKGROUND}

Throughout, $\Delta=\{z \in \mathbb{C}:|z|<1\}$ and $\mathbb{T}=\partial \Delta$. For $X$ and $Y$ complex Banach spaces, $\mathcal{L}(X, Y)$ denotes the space of continuous linear maps from $X$ to $Y, X^{\prime}=\mathcal{L}(X, \mathbb{C})$ and $\mathcal{L}(X)=\mathcal{L}(X, X)$. For $D$ a domain in $X, \mathcal{H}(D, Y)$ denotes all holomorphic maps from $D$ to $Y$ and $C(\bar{D}, Y)$ denotes continuous maps from $\bar{D}$ to $Y$.

Definition 1.1. A $J B^{*}$-triple is a complex Banach space $Z$ with a real trilinear mapping $\{\cdot, \cdot, \cdot\}: Z \times Z \times Z \rightarrow Z$ satisfying

(i) $\{x, y, z\}$ is complex linear and symmetric in the outer variables $x$ and $z$, and is complex anti-linear in $y$,

(ii) The map $z \mapsto\{x, x, z\}$, denoted $x \square x$, is Hermitian, $\sigma(x \square x) \geq$ 0 and $\|x \square x\|=\|x\|^{2}$ for all $x \in Z$, where $\sigma$ denotes the spectrum,

(iii) The product satisfies the Jordan triple identity, namely

$$
\{a, b,\{x, y, z\}\}=\{\{a, b, x\}, y, z\}-\{x,\{b, a, y\}, z\}+\{x, y,\{a, b, z\}\} .
$$

Throughout $Z$ will be a $J B^{*}$-triple and $B$ its open unit ball. 
The Jordan triple identity (1) implies that $i x \square x$ is a triple product derivation and it follows that $\exp i x \square x$ is a triple product automorphism. As $x \square x$ is Hermitian, $\exp i x \square x$ is then both a triple automorphism and a surjective linear isometry. In fact, a bijective linear map on a $J B^{*}$-triple is an isometry if, and only if, it is a triple homomorphism 8].

The triple product is continuous, $\|\{x, y, z\}\| \leq\|x\|\|y\|\|z\|$ [4] and $\|\{x, x, x\}\|=\|x\|^{3}$. Odd powers of an element $x$ exist, with $x^{(2 n+1)}:=$ $\left\{x, x^{(2 n-1)}, x\right\}, n \in \mathbb{N}, n \geq 1$, allowing us to define $p(x)$, for any odd polynomial $p$, leading to an odd functional calculus. The triple spectrum $K_{x} \subset[0,\|x\|]$ of the element $x \in Z$ is defined as $\left\{t \in \mathbb{R}^{+}: t^{2} \in\right.$ $\sigma(x \square x)\}$ [9]. The smallest closed subtriple of $Z$ containing $x$, denoted $Z_{x}$, is triple isomorphic to the commutative $\mathrm{C}^{*}$-algebra $C_{0}\left(K_{x}\right)$ via a linear map $j_{x}$ which takes $x$ to the identity function on $K_{x}$. In particular, if $p$ is an odd polynomial then $j_{x} \circ p=p \circ j_{x}$. We refer to $C_{0}\left(K_{x}\right)$ as the local structure of $Z$ at $x$.

There are natural linear maps $x \square y \in \mathcal{L}(Z): z \mapsto\{x, y, z\}, Q_{x} \in$ $\mathcal{L}_{\mathbb{R}}(Z): z \mapsto\{x, z, x\}$, and the Bergmann operators $B(x, y)=I-$ $2 x \square y+Q_{x} Q_{y} \in \mathcal{L}(Z)$.

Example 1.2. $\mathcal{L}(H, K)$, for complex Hilbert spaces $H$ and $K$, is a $J B^{*}$-triple with $\{x, y, z\}=\frac{1}{2}\left(x y^{*} z+z y^{*} x\right)$, where $y^{*}$ denotes the usual adjoint of $y$.

1.1. Algebraic identities. The Bergmann operators defined above play an important role in constructing quasi-inverses in a $J B^{*}$-triple. Given $x, y \in Z$, we say $(x, y)$ is a quasi-invertible pair, or that the quasi-inverse $x^{y}$ exists, if the Bergmann operator $B(x, y)$ is invertible in $\mathcal{L}(Z)$ and then the quasi-inverse $x^{y}$ is clefined to be $B(x, y)^{-1}\left(x-Q_{x}(y)\right)$. We note that $B(x, y)$ is invertible if, and only if, $B(y, x)$ is invertible. Invertibility of $B(x, y)$ always holds when $x, y \in B$ (indeed, more generally, when $\|x \square y\|<1$ ) and then $x^{y}=$ $(I-x \square y)^{-1} x=\sum_{k=0}^{\infty}(x \square y)^{k} x$. The quasi-inverse is a crucial component in the algebraic expression of the biholomorphic automorphisms of the unit ball (see below).

The algebraic identities listed in [12, Appendix A] for finite dimensional Jordan pairs are valid for general $J B^{*}$-triples. We recall 
several for later convenience:

$$
\begin{aligned}
B(x, y+z) & =B(x, y) B\left(x^{y}, z\right) \\
B(y+z, x) & =B\left(z, x^{y}\right) B(y, x) \\
B(x, y)^{-1} & =B\left(x^{y},-y\right) \\
B\left(B(u, v) x, B(v, u)^{-1} y\right) & =B(u, v) B(x, y) B(v, u)^{-1} \\
x^{y+z} & =\left(x^{y}\right)^{z} \\
(x+z)^{y} & =x^{y}+B(x, y)^{-1} z^{\left(y^{x}\right)} \\
(B(x, y) z)^{y} & =B(x, y) z^{B(y, x) y}
\end{aligned}
$$

For $a \in B$, the Bergmann operator $B(a, a)$ has positive spectrum and a unique square root $B(a, a)^{\frac{1}{2}}$ with positive spectrum which we will denote by $B_{a}$. Via the functional calculus and local structure, the use of the identities above can be somewhat extended. For example, $\mathbb{P 3 6}$ may be used with the operator $B_{a}=B(a, a)^{\frac{1}{2}}$ in place of $B(u, v)$; that is $B\left(B_{a} x, B_{a}^{-1} y\right)=B_{a} B(x, y) B_{a}^{-1}$. The local structure also allows one to make certain calculations in a commutative setting such as

$$
a^{a}=B_{a}^{-1} a .
$$

1.2. Automorphisms. The defining characteristic of the unit ball $B$ of a $J B^{*}$-triple $Z$ is its transitivity under the group of biholomorphic mappings $\operatorname{Aut}(B)$, ensuring that $B$ is a bounded symmetric domain. The algebraic characterisation of all bounded symmetric domains [8] yields Definition 1.1 and an explicit description ([8, 4.6]) of the elements of $\operatorname{Aut}(B)$. To be precise, every $g \in \operatorname{Aut}(B)$ can be written in the form $g=T g_{a}$, where $T$ is a surjective linear isomety of $Z$ and $g_{a}$ is a generalised Möbius map, or transvection, defined on $B$ by

$$
g_{a}(x)=a+B_{a} x^{-a} .
$$

Evidently, $g_{a}(0)=a$ and one can show that $g_{a}^{-1}=g_{-a}$. (This factorisation of $g=T g_{a}$ is unique for if $T g_{a}=S g_{b}$ then $g_{-b} S^{-1} T=g_{-a}$ and applying to the origin yields $a=b$ and then $S=T$.) For $a \in B$, the quasi-inverse $x^{-a}$, and hence $g_{a}$ and every element of Aut $(B)$, is defined and continuous beyond the unit ball, to the ball of radius $\|a\|^{-1}$. The quasi-inverse map $x \mapsto x^{y}$ is holomorphic on its domain and its derivative at $x_{0}$ is given by $B\left(x_{0}, y\right)^{-1}$ (see [12]). It follows then that the derivative of $g_{a}$ at $x_{0}$ is $B_{a} B\left(x_{0},-a\right)^{-1}$ and so $g_{a}^{\prime}(0)=B_{a}$ while $g_{a}^{\prime}(-a)=B_{a}^{-1}$. Note that $T g_{a}=g_{T a} T^{-1}$ so we may also choose to write $g \in \operatorname{Aut}(B)$ uniquely in the form $g=g_{b} S$. In this case, $b=g(0)$. 
1.3. Tripotents. A tripotent is an element $e \in Z$ satisfying $\{e, e, e\}=$ $e$ and, since $\|\{e, e, e\}\|=\|e\|^{3}$, any non-zero tripotent is a unit vector. Every non-zero tripotent $e$ induces a splitting of $Z$, as $Z=$ $Z_{0}(e) \oplus Z_{\frac{1}{2}}(e) \oplus Z_{1}(e)$, where $Z_{\lambda}(e)$ is the $\lambda$-eigenspace of $e \square e$. Mutually orthogonal projections of $Z$ onto $Z_{0}(e), Z_{\frac{1}{2}}(e)$, and $Z_{1}(e)$ are given by $P_{0}(e)=B(e, e), P_{\frac{1}{2}}(e)=2\left(e \square e-Q_{e} Q_{e}\right)$ and $P_{1}(e)=Q_{e} Q_{e}$, respectively .

A tripotent is called maximal if $Z_{0}(e)=\{0\}$ or, equivalently, if $B(e, e)=0$ and is called unitary if $Z_{1}(e)=Z$, that is, $P_{1}(e)=Q_{e} Q_{e}=$ $I$. For any $J B^{*}$-triple, the set of real extreme points of $\bar{B}$, the set of complex extreme points of $\bar{B}$ and the set of maximal tripotents all coincide. For details see [12].

Let $\Gamma$ be the set of maximal tripotents and $\Gamma_{1}$ be the set of unitary tripotents of the $J B^{*}$-triple $Z$. If $B(a, a)=0$ or $Q_{a} Q_{a}=I$ then consideration of $0=B(a, a) a$, or $a=Q_{a}^{2} a$ in the local triple $Z_{a}$ shows $a$ to be a tripotent and so:

Lemma 1.3. $\Gamma=\{a \in \bar{B}: B(a, a)=0\}$ and $\Gamma_{1}=\left\{a \in \bar{B}: Q_{a} Q_{a}=I\right\}$.

An important consequence of Lemma 1.3 is that both $\Gamma$ and $\Gamma_{1}$ are closed. We say $x, y \in Z$ are orthogonal, $x \perp y$, if $x \square y=0$ (equivalently $y \square x=0$ ). In particular, if $c$ and $e$ are orthogonal tripotents then $c+e$ is also a tripotent. This gives a partial ordering on the set, $M$, of all tripotents in $Z$ as follows.

Definition 1.4. For tripotents $c$ and $e$ we say $c<e$ if $e-c \in M$ and $(e-c) \perp c$.

Maximality of a tripotent with respect to this ordering is consistent with the notion of maximal tripotent given previously in the sense that a maximal tripotent is order maximal. A tripotent $e$ is minimal if $Z_{1}(e)=\mathbb{C} e . Z$ is said to have finite rank $r$ if every element $z \in Z$ is contained in a subtriple of (complex) dimension $\leq r$, and $r$ is minimal with this property. We say $x$ is a rank $k$ element if $Z_{x}$ is $k-$ dimensional. The rank one $J B^{*}$-triples are the Hilbert spaces. Other finite rank examples are sub-triples of $\mathcal{L}(H, K)$ where either $H$ or $K$ is finite dimensional, and spin factors. If $Z$ has finite rank $r$, a frame is a set $\left\{e_{1}, \ldots, e_{r}\right\}$ of non-zero pairwise orthogonal minimal tripotents and every $z \in Z$ has a unique spectral decomposition, called its Peirce decomposition, as $z=\lambda_{1} e_{1}+\cdots+\lambda_{r} e_{r}$, for some frame $\left\{e_{1}, \ldots, e_{r}\right\}$ and scalars $0 \leq \lambda_{1} \leq \ldots \leq \lambda_{r}=\|z\|$. See [2, 7] for details.

A $J B^{*}$-triple may not have any tripotents but if it is a dual Banach space (for example, the bidual of a $J B^{*}$-triple is a $J B^{*}$-triple [1]) then 
the Krein-Millman theorem implies the existence of maximal tripotents. Finite rank $J B^{*}$-triples have maximal tripotents and indeed are reflexive Banach spaces.

\section{INVARIANCE UNDER $g \in \operatorname{Aut}(B)$}

As mentioned above, biholomorphic automorphisms of the open unit ball extend continuously to a neighbourhood of $\bar{B}$. These maps do not, however, preserve the set of tripotents generally.

Example 2.1. The commutative $\mathrm{C}^{*}$-algebra, $\mathbb{C}^{2}$, is a $J B^{*}$-triple via the product $\{f, g, h\}=f \bar{g} h$ (corresponding to the maximum norm). Take $e$ to be the tripotent $(1,0)$ and $a=\left(\frac{1}{2}, \frac{1}{2}\right)$ and notice that for $g_{a} \in \operatorname{Aut}(B), g_{a}(e)=\left(1, \frac{1}{2}\right)$ is not a tripotent.

Nonetheless, the automorphisms of $B$ do act invariantly both on the set of maximal tripotents and on the set of unitary tripotents (when these are non-empty). In order to prove this we require the following results establishing key identities involving the Bergmann operators.

Proposition 2.2. Let $a \in B$ and $b \in \bar{B}$. Then

$$
B\left(g_{a}(b), g_{a}(b)\right)=B_{a} B(b,-a)^{-1} B(b, b) B(-a, b)^{-1} B_{a} .
$$

Proof. Recalling that $g_{a}(b)=a+B_{a} b^{-a}$ we proceed to expand as follows.

$$
\begin{aligned}
B\left(g_{a}(b), g_{a}(b)\right) & =B\left(a+B_{a} b^{-a}, a+B_{a} b^{-a}\right) \\
\stackrel{\text { IP344 }}{=} & \underbrace{B\left(B_{a} b^{-a},\left(a+B_{a}\left(b^{-a}\right)\right)^{a}\right)}_{R} \cdot \underbrace{B\left(a, a+B_{a}\left(b^{-a}\right)\right)}_{S}
\end{aligned}
$$

Focusing on the Bergmann operator $R$, we have

$$
\begin{aligned}
\left(a+B_{a}\left(b^{-a}\right)\right)^{a} & \stackrel{\text { एPA2 }}{=} a^{a}+B(a, a)^{-1}\left(B_{a}\left(b^{-a}\right)\right)^{\left(a^{a}\right)} \\
& \stackrel{\text { 2 }}{=} B_{a}^{-1}\left[a+B_{a}^{-1}\left(B_{a}\left(b^{-a}\right)\right)^{B_{a}^{-1} a}\right] \\
& \stackrel{\text { IPS }}{=} B_{a}^{-1}\left[a+B_{a}^{-1} B_{a}\left(b^{-a}\right)^{a}\right]=B_{a}^{-1}(a+b)
\end{aligned}
$$


and consequently

$$
\begin{aligned}
& R=B\left(B_{a} b^{-a}, B_{a}^{-1}(a+b)\right) \\
& \stackrel{\text { IP36 }}{=} B_{a} B\left(b^{-a}, a+b\right) B_{a}^{-1} \\
& \stackrel{\text { IP33 }}{=} B_{a} B\left(b^{-a}, a\right) B(b, b) B_{a}^{-1} \\
& \stackrel{\text { IP35 }}{=} B_{a} B(b,-a)^{-1} B(b, b) B_{a}^{-1} .
\end{aligned}
$$

A similar expansion gives

$$
S=B_{a} B(-a, b)^{-1} B_{a}
$$

and we are done.

Lemma 2.3. Let $a, b \in B$. Then

(i) $k(a, b)=B_{g_{a}(b)}^{-1} B_{a} B(b,-a)^{-1} B_{b}$ is a surjective linear isometry of $Z$,

(ii) $g_{a} g_{b}=g_{g_{a}(b)} k(a, b)$.

Proof. Consider the biholomorphic map on $B$ given by $k(a, b)=$ $g_{-g_{a}(b)} g_{a} g_{b}$. Since this map fixes 0 , the Schwarz Lemma guarantees it is a linear isometry which agrees with its derivative at the origin and this, via the chain rule, is given by

$$
g_{-g_{a}(b)}^{\prime}\left(g_{a}(b)\right) \cdot g_{a}^{\prime}(b) \cdot g_{b}^{\prime}(0)=B_{g_{a}(b)}^{-1} B_{a} B(b,-a)^{-1} B_{b} .
$$

We now provide proofs of the invariance of the set of maximal tripotents $\Gamma$, and the set of unitary tripotents $\Gamma_{1}$ under automorphisms. The result for $\Gamma$ is effectively provided in [5, 2.4(i)] using the characterisation of maximal tripotents as complex extreme points. Here we present an algebraic proof which follows immediately from Proposition 2.2.

Theorem 2.4. For $g \in \operatorname{Aut}(B), g(\Gamma)=\Gamma$, if $\Gamma \neq \emptyset$.

Proof. Write $g=T g_{a}$ where $T$ is a surjective linear isometry and $g_{a}$ is a Möbius map. Surjective linear isometries (i.e. triple product automorphisms) not only preserve tripotents, but also preserve the maximality of a tripotent. Indeed, $f \in \bar{B}$ is a maximal tripotent precisely when $B(f, f)=0$ and, as $T$ is a triple automorphism, this coincides with $T B(f, f) T^{-1}=B(T f, T f)$ vanishing and $T f$ being a maximal tripotent.

Thus we must show that for $a \in B$ and a maximal tripotent $e$, we have $g_{a}(e)$ is a maximal tripotent. Since $e$ is a maximal tripotent, $B(e, e)=0$ and from Proposition 2.2 we have $B\left(g_{a}(e), g_{a}(e)\right)=0$ 
and thus $g_{a}(e)$ is a maximal tripotent. This proves inclusion, while equality follows by invertibility and $g_{a}^{-1}=g_{-a}$.

Proposition 2.5. Let $a, b \in B$. Then $g_{a}(b)=\widetilde{k} g_{b}(a)$, where $\widetilde{k}$ is $a$ surjective linear isometry.

Proof. By inversion of Lemma 2.3)(ii), we can say

$$
g_{-b} g_{-a}=k(a, b)^{-1} g_{-g_{a}(b)}=k(a, b)^{-1} g_{g_{-a}(-b)}
$$

and swapping the roles of $a$ and $-b$, we have

$$
g_{a} g_{b}=k(-b,-a)^{-1} g_{g_{b}(a)} .
$$

Comparison with Lemma 2.3(ii) yields $g_{g_{a}(b)} k(a, b)=k(b, a)^{-1} g_{g_{b}(a)}$. Apply this to the origin to gain the result, together with $\widetilde{k}=k(b, a)^{-1}$.

Theorem 2.6. For $g=\in \operatorname{Aut}(B), g\left(\Gamma_{1}\right)=\Gamma_{1}$, if $\Gamma_{1} \neq \emptyset$.

Proof. Again write $g=T g_{a}$ where $T$ is a surjective linear isometry and $g_{a}$ is a Möbius map. As in the proof for maximal tripotents, invariance with respect to the linear part $T$ is immediate and we need only show that the image of a unitary tripotent under the transvection $g_{a}$ is a unitary tripotent. This equates to proving $Q_{g_{a}(u)} Q_{g_{a}(u)}=I$ when $Q_{u} Q_{u}=I$.

Let $t \in(0,1)$ so that $t u \in B$. Proposition 2.5 allows us to write $g_{a}(t u)=k_{t} g_{t u}(a)$, for some surjective linear isometry $k_{t}$. As $k_{t}$ is a triple automorphism it follows that $Q_{g_{a}(t u)}=Q_{k_{t} g_{t u}(a)}=k_{t} Q_{g_{t u}(a)} k_{t}^{-1}$ and so

$$
I-Q_{g_{a}(t u)} Q_{g_{a}(t u)}=k_{t}\left(I-Q_{g_{t u}(a)} Q_{g_{t u}(a)}\right) k_{t}^{-1} .
$$

In particular,

$$
\left\|I-Q_{g_{a}(t u)} Q_{g_{a}(t u)}\right\|=\left\|I-Q_{g_{t u}(a)} Q_{g_{t u}(a)}\right\| .
$$

Being unitary, the tripotent $u$ is also maximal and the Bergman operator $B(u, u)=0$. Triple product continuity implies then that $B(t u, t u) \rightarrow 0$. This convergence passes to the square root. Indeed, by [10, Lemma 3.4], $\left\|B_{t u}\right\|=\left\|B_{u}-B_{t u}\right\| \leq 2 \sqrt{1-t^{2}}$ and so $B_{t u} \rightarrow 0$ as $t \rightarrow 1$. Since $a^{-t u} \rightarrow a^{-u} \in Z$, we can say that $g_{t u}(a)=t u+B_{t u} a^{-t u} \rightarrow u$ as $t \rightarrow 1$. Again from continuity of the triple product, $Q_{g_{t u}(a)} \rightarrow Q_{u}$ and hence $\left\|I-Q_{g_{t u}(a)} Q_{g_{t u}(a)}\right\| \rightarrow 0$. Combine this with (3) and the convergence of $g_{a}(t u)$ to $g_{a}(u)$ to conclude that $Q_{g_{a}(u)} Q_{g_{a}(u)}=I$ as required. 


\section{RusSO-DYE TYPE RESULTS FOR JB*-TRIPLES}

The classical Russo-Dye Theorem states that the closed unit ball of a unital $C^{*}$-algebra is the closed convex hull of its extreme points [15]. Variations of the second statement in Theorem 3.1 below appear in [13] and [16, but here we do not require that $Z$ is a JBW*-triple (i.e. has a predual). We also provide a unified proof of both statements below, based on a technique used by Harris [6].

Theorem 3.1. Let $Z$ be a J $B^{*}$-triple with open unit ball $B$.

(1) If $Z$ contains a maximal tripotent then $\bar{B}=\overline{\mathrm{co}}(\Gamma)$.

(2) If $Z$ contains a unitary tripotent then $\bar{B}=\overline{\mathrm{co}}\left(\Gamma_{1}\right)$

Proof. Let $b \in B$. For (1), choose $a \in \Gamma$ and, respectively for (2), $a \in \Gamma_{1}$. Define $h(\lambda)=g_{b}(\lambda a)$ to gain a $Z$-valued holomorphic function on the disc of radius $\frac{1}{\|b\|}>1$ in $\mathbb{C}$. The mean value property for holomorphic functions implies

$$
\begin{aligned}
b=h(0) & =\frac{1}{2 \pi} \int_{0}^{2 \pi} h\left(e^{i \theta}\right) d \theta \\
& =\frac{1}{2 \pi} \int_{0}^{2 \pi} g_{b}\left(e^{i \theta} a\right) d \theta .
\end{aligned}
$$

For each $\theta \in \mathbb{R}, e^{i \theta} a \in \Gamma$ (resp. $\Gamma_{1}$ ) and, by Theorem 2.4 (resp. Theorem 2.6), so is $g_{b}\left(e^{i \theta} a\right)$. Thus $b \in \overline{\mathrm{co}}(\Gamma)\left(\right.$ resp. $\left.\overline{\mathrm{co}}\left(\Gamma_{1}\right)\right)$ as stated.

In fact, more general Russo-Dye extensions are possible. For any $v \in \partial B$, we let $G_{v}:=\operatorname{Aut}(B)(v)$ be the orbit of $v$ under the automorphisms of $B$ and we let $G_{v}^{0}:=\operatorname{Aut}(B)^{0}(v)$ be the orbit of $v$ under the automorphisms in $\operatorname{Aut}(B)^{0}$, the connected component of the identity in Aut $(B)$. Note that every Möbius map $g_{a}$ and every unimodular rotation $e^{i \theta}$ lies in Aut $(B)^{0}$. Replacing $a$ in the above proof with $v \in \partial B$, means $g_{b}\left(e^{i \theta} v\right) \in G_{v}^{0}$, for all $b \in B$ and $\theta \in \mathbb{R}$ and yields the following.

Corollary 3.2. Let $Z$ be a JB*-triple with open unit ball B. For any $v \in \partial B$,

$$
\bar{B}=\overline{\mathrm{co}}\left(G_{v}^{0}\right)=\overline{\mathrm{co}}\left(G_{v}\right) .
$$

We will see later in Corollary 5.12 that if $Z$ is a finite rank triple then $\Gamma \subseteq \overline{G_{v}^{0}}$, for all $v \in \partial B$. 


\section{BOUNDARY SUBSETS THAT ARE DETERMINING FOR HOLOMORPHIC}

FUNCTIONS

An immediate Corollary of Theorem 3.1 is the result below (and equation (4) in particular) showing that holomorphic functions on $B$ having a continuous extension to $\bar{B}$ are already determined by their values on $\Gamma$ or $\Gamma_{1}$, when these are non-empty. This can be described by saying that the sets $\Gamma$ and $\Gamma_{1}$ are determining for holomorphic functions on $B$. In fact, there are many such determining sets in the boundary of a bounded symmetric domain but for clarity we begin with $\Gamma$ and $\Gamma_{1}$.

Proposition 4.1. Let $Z$ be a JB*-triple with open unit ball $B$ and $X$ be any Banach space. Let $f: B \mapsto X$ be holomorphic on $B$ with a continuous extension to $\bar{B}$.

(1) If $Z$ contains a maximal tripotent then $f(\bar{B}) \subseteq \overline{\mathrm{CO}}(f(\Gamma))$ and

$$
\sup \{\|f(z)\|: z \in \bar{B}\}=\sup \{\|f(z)\|: z \in \Gamma\} .
$$

(2) If $Z$ contains a unitary tripotent then $f(\bar{B}) \subseteq \overline{\mathrm{co}}\left(f\left(\Gamma_{1}\right)\right)$ and

$$
\sup \{\|f(z)\|: z \in \bar{B}\}=\sup \left\{\|f(z)\|: z \in \Gamma_{1}\right\} .
$$

Proof. The two versions have similar proof, so we only present that of (1). Suppose then that $\Gamma \neq \emptyset$ and then let $a \in \Gamma$. Let $b \in B$ be arbitrary. Applying the proof of Theorem 3.1 to the map $h(\lambda)=f\left(g_{b}(\lambda a)\right)$ and using the mean value property gives

$$
f(b)=\frac{1}{2 \pi} \int_{0}^{2 \pi} f\left(g_{b}\left(e^{i \theta} a\right)\right) d \theta .
$$

It follows that

$$
\|f(b)\| \leq \frac{1}{2 \pi} \int_{0}^{2 \pi} \sup \{\|f(z)\|: z \in \Gamma\} d \theta=\sup \{\|f(z)\|: z \in \Gamma\}
$$

and we are done.

Remarks 4.2. We note that, unlike in finite dimensions, $\sup \{\|f(z)\|$ : $z \in \bar{B}\}$ may not actually be achieved. However, the above means that if $f$ is bounded on $\Gamma$ or $\Gamma_{1}$, it must also be bounded on $\bar{B}$ and thus, if $f$ is unbounded then all suprema above must be infinite.

Just as Theorem 3.1 leads to Proposition 4.1 we have the following from Corollary 3.2 
Proposition 4.3. Let $Z$ be a J $B^{*}$-triple with open unit ball $B$ and $X$ be any Banach space. Let $f: B \mapsto X$ be holomorphic on $B$ with a continuous extension to $\partial B$. For any $v \in \partial B$ we have

$$
f(\bar{B}) \subseteq \overline{\operatorname{co}} f\left(G_{v}^{0}\right)
$$

and

$$
\sup \{\|f(z)\|: z \in \bar{B}\}=\sup \left\{\|f(z)\|: z \in G_{v}^{0}\right\} .
$$

In particular, if $f$ is unbounded then both suprema are infinite.

We recall that if a $J B^{*}$-triple has finite rank, then the boundary of its unit ball is the disjoint union of holomorphic boundary components defined as follows.

Definition 4.4. 10, 4.1] A non-empty set $A \subset \bar{B}$ is a holomorphic boundary component of $B$ if $A$ is minimal with respect to the fact that, for all $f \in \mathcal{F}=\{f: \Delta \rightarrow Z$ holomorphic with $f(\Delta) \subset \bar{B}\}$, either

$$
f(\Delta) \subset A \text { or } f(\Delta) \subset \bar{B} \backslash A \text {. }
$$

By replacing $\mathcal{F}$ in the above definition with the set of all complex affine maps : $\Delta \rightarrow \bar{B}$ we get the definition of (complex) affine boundary components.

Remarks 4.5. It follows that if $D$ is a domain and $g: D \rightarrow \bar{B}$ is a holomorphic map then $g(D)$ must lie in a single such boundary component.

The following shows that holomorphic and affine boundary components coincide in the finite rank case and each is determined by a unique tripotent.

Theorem 4.6. [10, 4.2, 4.3, 4.4] Let $Z$ be a finite rank JB*-triple with open unit ball $B$. The following hold.

(i) Holomorphic and affine boundary components coincide and are precisely the sets

$$
K_{e}=e+B_{0}(e)
$$

where $e$ is a tripotent and $B_{0}(e)=B \cap Z_{0}(e)=P_{0}(e)(B)$.

(ii) The map $e \rightarrow K_{e}$ is a bijection between the set, $M$, of tripotents in $Z$ and the set of boundary components of $B$, with $x \in K_{e}$ if, and only if, $e=\lim _{n \rightarrow \infty} x^{(2 n+1)}$.

(iii) $\bar{K}_{e}=\bigcup_{d \geq e} K_{d}$.

Henceforth we refer simply to boundary component and write $K_{x}$ for the boundary component of $x$. We note that $x$ is an extreme 
point if, and only if, $K_{x}=\{x\}$. Indeed, if a boundary component $K_{x}$ contains an extreme point $v$ then $K_{x}=\{v\}$.

Definition 4.7. We define the rank of a boundary component $K_{e}$ in a finite rank triple $Z$ as the rank of the $\mathrm{JB}^{*}$-subtriple $Z_{0}(e)$ (with rank zero if $\left.Z_{0}(e)=\{0\}\right)$.

Since $K_{e}=e+B_{0}(e)$, where $B_{0}(e)=B \cap Z_{0}(e)$, then $K_{e}$ is biholomorphically equivalent to the bounded symmetric domain $B_{0}(e)$, which is the open unit ball of the $J B^{*}$-triple $Z_{0}(e)$. In other words, the rank of the boundary component $K_{e}$ in the sense of Definition (4.7) is prescisely its rank as a bounded symmetric domain. Moreover, if a tripotent $e$ is a rank $k$ element $\left(Z_{e}\right.$ is $k$ dimensional), then its boundary component $K_{e}$ is rank $n-k$. This means that if $Z$ is rank $n$ then it has boundary components of rank $k \in\{0,1, \ldots, n\}$. The only rank $n$ boundary component is $B\left(=K_{0}\right)$ itself, the rank zero components are singletons given by the maximal extreme points for which $B_{0}(e)=\{0\}$, and there are components of all rank $k \in\{0,1, \ldots, n\}$.

Theorem 2.4 which underpins the Russo-Dye extensions in Theorem 3.1 and Corollary 3.2. showed that the extreme points, or rank zero boundary components, are invariant under elements of Aut $(B)$. In fact, Theorem 2.4 is a special case of a more general result, namely, that every automorphism of $B$ maps rank $k$ boundary components onto rank $k$ boundary components, for all $k \in\{0,1, \ldots, n\}$. We note that this holds true despite the fact that automorphisms do not generally preserve the rank of individual elements, nor must automorphisms even map tripotents to tripotents, as seen in Example 2.1 above.

For $g \in \operatorname{Aut}(B)$, we recall that $g: \partial B \mapsto \partial B$ and since $g$ extends to a holomorphic map on an open neighbourhood of $\bar{B}$, it follows that $\left.g\right|_{K_{v}}$ is holomorphic on $K_{v}$.

Proposition 4.8. Let $Z$ be a finite rank $J B^{*}$-triple. Let $v \in \bar{B}$ and $g \in \operatorname{Aut}(B)$. Then

(1) $g\left(K_{v}\right)=K_{g(v)}$;

(2) $\operatorname{rank}\left(K_{v}\right)=\operatorname{rank}\left(K_{g(v)}\right)$.

Proof. Let $v \in \bar{B}$ be arbitrary and $K_{v}$ be its boundary component. Case (i): Suppose $v$ is not extreme, so $K_{v}$ is biholomorphically equivalent to a non-trivial domain. Then $g\left(K_{v}\right) \cap K_{g(v)} \neq \emptyset$ (as it contains $g(v)$ ), so from Remarks $4.5 g\left(K_{v}\right) \subseteq K_{g(v)}$. Then $K_{g(v)}$ is not a singleton, so $g(v)$ is not extreme, and the above argument applied to $g(v)$ 
and $g^{-1}$ gives $g^{-1}\left(K_{g(v)}\right) \subseteq K_{v}$. Thus $g\left(K_{v}\right)=K_{g(v)}$.

Case (ii): Let $v$ be extreme. Then $g(v) \in K_{u}$, for some tripotent $u$ and if $u$ is not extreme, applying (i) to $u$ and $g^{-1}$ gives $g^{-1}\left(K_{u}\right)=K_{g^{-1}(u)}$. Then $v \in K_{g^{-1}(u)}$ is extreme, so $\{v\}=K_{g^{-1}(u)}=g^{-1}\left(K_{u}\right)$. This is impossible as $g^{-1}$ is injective and $K_{u}$ is a non-trivial domain. In other words, $v$ extreme implies $u$ extreme and hence $g(v)$ is extreme, completing (1) above.

To prove (2). Let $v \in \bar{B}$. From (ii), $v$ is extreme if, and only if, $g(v)$ is extreme, in which case, $\operatorname{rank}\left(K_{v}\right)=\operatorname{rank}\left(K_{g(v)}\right)=0$. We assume therefore that $v, g(v)$ are not extreme. There exist tripotents $e, f$ with (and from (1))

$$
K_{v}=K_{e}=e+B_{0}(e) \text { and } g\left(K_{v}\right)=K_{g(v)}=K_{f}=f+B_{0}(f),
$$

where $B_{0}(e)$ and $B_{0}(f)$ are the open units balls of (non-zero) $J B^{*}$ triples $Z_{0}(e)$ and $Z_{0}(f)$ (respectively). We define

$$
h: B_{0}(e) \mapsto B_{0}(f) \text { given by } h(z)=g(z+e)-f \text {. }
$$

Clearly, $h$ is holomorphic with holomorphic inverse

$$
h^{-1}: B_{0}(f) \mapsto B_{0}(e) \text { given by } h^{-1}(w)=g^{-1}(w+f)-e .
$$

In other words, the open unit balls of $Z_{0}(e)$ and $Z_{0}(f)$ are biholomorphically equivalent (under $h$ ). By [11], two Banach spaces are linearly isometric if, and only if, their open unit balls are biholomorphically equivalent, so $Z_{0}(e)$ and $Z_{0}(f)$ are linearly isometric. A linear isometry of triples preserves the triple rank, so $Z_{0}(e)$ and $Z_{0}(f)$ have the same rank as triples and hence $K_{v}=K_{e}$ and $K_{g(v)}=K_{f}$ have the same rank as boundary components.

Corollary 4.9. Let $Z$ be a JB*-triple of finite rank $n$. For each $k \in$ $\{0, \ldots, n-1\}$ there is a determining set in $\partial B$ whose points all lie in rank $k$ boundary components.

Proof. Fix $0 \leq k \leq n-1$. Choose any tripotent $e$ of rank $n-k$. The boundary component $K_{e}$ has rank $k$. Pick any $v \in K_{e}$. Proposition 4.8 above proves that for all $g \in G=\operatorname{Aut}(B), \operatorname{rank}\left(K_{g(v)}\right)=\operatorname{rank}\left(K_{v}\right)$. In other words, each element in $G_{v}$ (and hence in $G_{v}^{0}$ ) lies in a rank $k$ boundary component.

We note this does not mean that $G_{v}^{0}$ or $G_{v}$ (for $v \in \partial B$ ) lies in any one boundary component. For example, if $k=0$, the rank $k$ boundary components are the extreme points in $\Gamma$, and this set is generally not connected in the infinite dimensional case. Also of relevance to later results is the fact that the determining sets $G_{v}$ above are not closed in general. To illustrate, let us return to Example 1.2 
Example 4.10. Take $v=\left(1, \frac{1}{2}\right)$ in the boundary of the unit ball of $Z=\mathbb{C}^{2}$ for the maximum norm. Then $G_{v}^{0}=\mathbb{T} \times \Delta$ while $G_{v}=$ $(\mathbb{T} \times \Delta) \cup(\Delta \times \mathbb{T})$. The holomorphic boundary component of $v$ is $K_{v}=\{1\} \times \Delta$, and this equals $K_{e}$ where $e=(1,0)$ is the unique tripotent in $K_{v}$. The closure of $G_{v}^{0}$ is a proper subset of the boundary which properly contains the set of extreme points $\Gamma=\mathbb{T} \times \mathbb{T}$. Each extreme point is not only a maximal tripotent, but also unitary. These last two facts will be reflected in Corollaries 5.11 and 5.12 .

We have shown that there are many determining sets in the boundary of a bounded symmetric domain, for example, $\Gamma_{1}, \Gamma, G_{v}, G_{v}^{0}$, for arbitrary $v \in \partial B$. Nonetheless, we will show that the role played by the set, $\Gamma$, of extreme points remains special. We recall that in finite dimensions, the Bergmann-Shilov boundary of $B$ is defined as the minimal closed subset of $\bar{B}$ on which every $f: \bar{B} \mapsto \mathbb{C}$ which is holomorphic on $B$ and continuous on $\bar{B}$ achieves its maximum modulus. Moreover, the Bergmann-Shilov boundary in finite dimensions is exactly the set of extreme points, $\Gamma$ [12. Theorem 6.5].

While Propositions 4.1 and 4.3 can already be viewed as partial extensions of Bergmann-Shilov type behaviour to infinite dimensions, in the next section we will prove that for finite rank triples, the set of extreme points $\Gamma$ is the key determining set and is exactly a Bergmann-Shilov boundary relative to such holomorphic functions.

\section{An infinite dimensional analogue of the Bergmann-Shilov BOUNDARY}

Let $Z$ be a finite rank $J B^{*}$-triple. We recall now an equivalent norm defined on $Z$ by means of the spectral decomposition [2. Section 9.2]. Namely, for $x \in Z$ we have $x=\alpha_{1} e_{1}+\cdots+\alpha_{n} e_{n}$, with $\|x\|=$ $\alpha_{1} \geq \alpha_{2} \cdots \geq \alpha_{n} \geq 0$, and $e_{1}, \cdots, e_{n}$ a frame of mutually orthogonal minimal tripotents. To each minimal tripotent, [3], $e_{i}$ there exists a unique $\phi_{i} \in Z_{*}$ (the unique predual of $Z$ ) such that $\phi_{i}$ is extreme in $\bar{B}_{Z_{*}}$ and $\phi_{i}\left(e_{i}\right)=1$. This allows us to define an inner product, often called the algebraic inner product, on $Z \times Z$ by $\langle x, y\rangle_{a}:=$ $\sum_{i=1}^{n} \alpha_{i} \overline{\phi_{i}(y)}$ and $\|x\|_{a}^{2}:=\langle x, x\rangle_{a}=\sum_{i=1}^{n} \alpha_{i}^{2}$ and

$$
\|x\|^{2} \leq\|x\|_{a}^{2} \leq n\|x\|^{2} .
$$

We quote the following version of the Maximum Modulus Principle [14. Cor. 2.2].

Theorem 5.1 (Maximum Modulus). Let $D$ be a domain in a complex Banach space $Z$ and $f \in \mathcal{H}(D, X)$, where $X$ is a Banach space. 
Then $f$ satisfies the maximum modulus principle, namely, if $\|f(z)\|$ achieves a maximum at any point of $D$ then $\|f(z)\|$ is constant.

Proposition 5.2. Let $Z$ be a finite rank JB*-triple, $X$ be a Banach space and $f$ be a holomorphic map on a neighbourhood of $\bar{B}$ into $X$. If $\|f(z)\|$ achieves a maximum on $\bar{B}$ then it must achieve this maximum on $\Gamma$.

Proof. Suppose $\|f(z)\|$ achieves a maximum $M$ at $w \in \bar{B}$. If $w \in B$ then by the maximum modulus principle above, $\|f(z)\|$ is constant on $B$ and hence on $\bar{B}$, thereby achieving its maximum at every element of $\Gamma$. Thus we may assume $w \in \partial B$. If $w$ is an extreme point we are done, so we may assume that $w$ is an interior point of its boundary component $K_{w}$.

Since $Z$ is finite rank, there is a unique tripotent $e$ such that $K_{w}=$ $K_{e}=e+\left(Z_{0}(e) \cap B\right)$. We define a holomorphic map $h$ on $D:=$ $Z_{0}(e) \cap B$ by $h(z)=f(e+z)$. Clearly, $\|h(z)\|$ is bounded by $M$ and achieves this bound at $z_{0} \in D$ where $w=e+z_{0}$. Again by Theorem 5.1 $\|h(z)\|$ is constant on $D$ and so $\|f(z)\|$ is constant on $K_{e}$ and $\|f(e)\|=\|f(w)\|=M$. By continuity, $\|f(z)\|$ is then constant on $\overline{K_{e}}$. As $\overline{K_{e}}=\cup_{d \geq e} K_{d},\|f(z)\|$ is also constant on $K_{d}$, where $d$ is any tripotent that majorises $e$. Using the spectral decomposition, we can construct a maximal tripotent $e^{\prime}$ that majorises $e$. By maximality, $K_{e^{\prime}}=\left\{e^{\prime}\right\}$ so we have $e^{\prime} \in \Gamma$ with $\left\|f\left(e^{\prime}\right)\right\|=\|f(e)\|=M$ as required.

The hypothesis in Proposition 5.2 that $f$ be holomorphic on a open neighbourhood of $\bar{B}$ was chosen to ensure that the mapping $h(z)=f(e+z)$ defined on $D=Z_{0}(e) \cap B$ is itself holomorphic; where $K_{e}=e+\left(Z_{0}(e) \cap B\right)$ is the boundary component on which $f$ achieves maximum norm. In finite dimensions, it suffices for $f: B \mapsto \mathbb{C}$ that $f \in \mathcal{H}(B, \mathbb{C}) \cap C(\bar{B}, \mathbb{C})$. In that case, we use compactness of $\bar{B}$ and uniform convergence of the maps $h_{n}(z)=f\left(\left(\frac{n-1}{n}\right) e+z\right)$, which are clearly now holomorphic on $B$, to argue that $h$ is holomorphic as it is a uniform limit of holomorphic functions. Of course, in infinite dimensions $\bar{B}$ is not compact so we need other tools. These tools consist of a different topology on the space of all maps $\mathcal{H}(B, \mathbb{C})$, known as the compact-open topology and denoted here by $\tau$, together with a suitable $\tau$ analogue of Montel's theorem and knowing that $\tau$ is complete. We therefore use the following results, cf. [14, Proposition 2.4 and Theorem 2.10].

Theorem 5.3. Let $X, Y$ be Banach spaces and $D$ be a domain in $X$. The space $\mathcal{H}(D, Y)$ is complete with respect to $\tau$. 
We write $\|f\|_{D}:=\sup \{\|f(z)\|: z \in D\}$.

Theorem 5.4. Let $X, Y$ be Banach spaces and $D$ be a domain in $X$. Then the set $\mathcal{F}_{M}=\left\{f \in \mathcal{H}(D, Y):\|f\|_{D} \leq M<\infty\right\}$ is relatively compact with respect to $\tau$ if, and only if, each orbit $\mathcal{F}_{M}(x)$ is relatively compact in $Y$, for $x \in D$.

Theorem 5.5. Let $Z$ be a finite rank $J B^{*}$-triple with ball $B$. Let $f: B \mapsto \mathbb{C}$ be holomorphic on $B$ with a continuous extension to $\partial B$. If $|f(z)|$ achieves a maximum on $\bar{B}$ then it must achieve this maximum modulus on $\Gamma$.

Proof. Assume $|f(z)|$ achieves a maximum $M$ at $w \in \bar{B}$ and repeat the proof of Proposition 5.2 The only part of that proof that requires adapting to $f \in \mathcal{H}(B, \mathbb{C}) \cap C(\bar{B}, \mathbb{C})$ is to prove that $h: D=Z_{0}(e) \cap$ $B \mapsto \mathbb{C}$ given by $h(z)=f(e+z)$ is holomorphic, where $w=e+z_{0}$, as before. For $n \in \mathbb{N}$, define the holomorphic map $h_{n}: D \mapsto \mathbb{C}$ by $h_{n}(z)=f\left(\left(\frac{n-1}{n}\right) e+z\right)$. Using terminology from Theorem 5.4 with $Y=$ $\mathbb{C}, \mathcal{F}_{M}(x) \subset B(0, M) \subset \mathbb{C}$ is bounded and is hence relatively compact in $\mathbb{C}$, for each $x \in D$. Theorem 5.4 therefore implies that $\mathcal{F}_{M} \subseteq$ $\mathcal{H}(D, \mathbb{C})$ is $\tau$-relatively compact in $\mathcal{H}(D, \mathbb{C})$. The sequence $\left(h_{n}\right)_{n}$ in $\mathcal{F}_{M}$ must therefore have a $\tau$-convergent subnet (indexed by $\alpha$, say) $\left(h_{n_{\alpha}}\right)_{\alpha}$, converging to a $\tau$-limit $k$. Theorem 5.3 then implies that $k \in \mathcal{H}(D, \mathbb{C})$. Since $\tau$-converence implies pointwise convergence, we have in particular that, for all $z \in D$,

$$
k(z)=\lim _{\alpha} h_{n_{\alpha}}(z)=\lim _{\alpha} f\left(\left(\frac{n_{\alpha}-1}{n_{\alpha}}\right) e+z\right) .
$$

Continuity of $f$ to $\partial B$ then gives $k(z)=f(e+z)=h(z)$. In other words, $h=k$ and therefore $h$ is holomorphic on $D$. The rest of the proof then continues exactly as in Proposition 5.2

It follows from Proposition 5.2. Theorem 5.4 and the proof of Theorem 5.5 that $\mathbb{C}$ in Theorem 5.5 can be replaced by any finite dimensional Banach space.

Corollary 5.6. Let $Z$ be a finite rank $J B^{*}$-triple with ball $B$ and $X$ be any finite dimensional Banach space. Let $f: B \mapsto X$ be holomorphic on $B$ with a continuous extension to $\partial B$. If $\|f(z)\|$ achieves a maximum on $\bar{B}$ then it must achieve this maximum on $\Gamma$.

The following result is adapted from [12, Theorem 6.5].

Proposition 5.7. Let $Z$ be a finite rank JB*-triple and $X$ be a Banach space. For $e \in \Gamma$ there exists a holomorphic map $h: Z \rightarrow X$ such that $h$ achieves its maximum norm on $\bar{B}$ only at $e$. 
Proof. Fix $e \in \Gamma$ and $v \in X$ with $\|v\|=1$. Let $\langle\cdot, \cdot\rangle_{a}$ clenote the algebraic inner product on $Z$ defined above. Define a holomorphic function $h: Z \rightarrow X$ by $h(z)=\frac{1}{2}\left(1+\frac{\langle z, e\rangle_{a}}{\langle e, e\rangle_{a}}\right) v$. For $z \in \bar{B}$,

$$
\|h(z)\|=\frac{1}{2}\left|1+\frac{\langle z, e\rangle_{a}}{\langle e, e\rangle_{a}}\right| \leq \frac{1}{2}\left(1+\frac{\left|\langle z, e\rangle_{a}\right|}{\langle e, e\rangle_{a}}\right) .
$$

The Cauchy-Schwarz inequality gives $\left|\langle z, e\rangle_{a}\right| \leq\|z\|_{a}\|e\|_{a}$ with equality if, and only if, $z=\gamma e$, for $\gamma \in \mathbb{C}$.

Since $e$ is maximal, $\|e\|_{a}=\sqrt{n}$ and by $5\|z\|_{a} \leq \sqrt{n}\|z\|$ giving

$$
\|z\|_{a}\|e\|_{a} \leq n \text { for all } z \in \bar{B} \text {. }
$$

Therefore $\left|\frac{\langle z, e\rangle_{a}}{\langle e, e\rangle_{a}}\right| \leq 1$ for all $z \in \bar{B}$ with equality if, and only if, we have equality in both (7) and (8), namely

$$
\left|\frac{\langle z, e\rangle_{a}}{\langle e, e\rangle_{a}}\right| \leq 1 \text { with equality if, and only if, } z=\gamma e \text {, for } \gamma \in \mathbb{T}=\partial \Delta \text {. }
$$

Since for $\mu \in \mathbb{T}, \frac{1}{2}|1+\mu|=1$ precisely when $\mu=1$, it follows from (6) and (9) that $\|h(z)\| \leq 1$ for all $z \in B$, with equality if, and only if, $z=$ $e$.

In the absence of finite dimensionality, the above result still allows that the function $h$ may have norm determined (though not attained) away from $e$. To address this we require the following Lemma.

Lemma 5.8. Let $H$ be an inner product space, $e, z \in H$ with $\|z\| \leq\|e\|$ and $\epsilon>0$. There exists $\delta>0$ such that $\left|1-\frac{\langle z, e\rangle}{\langle e, e\rangle}\right|<\delta$ implies $\|z-e\|<\epsilon$.

Proof. Write $z^{\perp}=z-\frac{\langle z, e\rangle}{\langle e, e\rangle} e$ so that $z=\frac{\langle z, e\rangle}{\langle e, e\rangle} e+z^{\perp}$ where $\left\langle z^{\perp}, e\right\rangle=0$. Then $\left\|z^{\perp}\right\|^{2}+\frac{|\langle z, e\rangle|^{2}}{\langle e, e\rangle}=\|z\|^{2} \leq\|e\|^{2}$ which implies

$$
\left\|z^{\perp}\right\| \leq\|e\| \sqrt{1-\frac{|\langle z, e\rangle|^{2}}{\langle e, e\rangle^{2}}}
$$

Now $\left|1-\frac{\langle z, e\rangle}{\langle e, e\rangle}\right|<\delta$ gives $1-\left|\frac{\langle z, e\rangle}{\langle e, e\rangle}\right|<\delta$ and $1-\left|\frac{\langle z, e\rangle}{\langle e, e\rangle}\right|^{2}<2 \delta$ so $\left\|z^{\perp}\right\|<\|e\| \sqrt{2 \delta}$. Finally

$$
\begin{aligned}
\|z-e\| & =\left\|\left(z-z^{\perp}\right)-e+z^{\perp}\right\| \\
& \leq\left\|\frac{\langle z, e\rangle}{\langle e, e\rangle} e-e\right\|+\left\|z^{\perp}\right\|<\delta\|e\|+\sqrt{2 \delta}\|e\|
\end{aligned}
$$


which is smaller than $\epsilon$ for $\delta>0$ sufficiently small.

Proposition 5.9. Let $Z$ be a finite rank JB*-triple and $X$ be a Banach space. For $e \in \Gamma$, there exists a holomorphic map $h: Z \rightarrow X$ such that $\|f\|_{\bar{B}}=1$ but $\|f\|_{\bar{B} \backslash B(e, \epsilon)}<1$ for $\epsilon>0$.

Proof. The same function $h$ provided in Proposition 5.7 suffices. Observe that for $\mu \in \bar{\Delta}=\bar{\Delta}(0,1)$ and $\eta \in(0,1)$ then $\frac{1}{2}|1+\mu|>1-\eta$ implies $\mu \in \bar{\Delta}(0,1) \backslash \Delta(-1,2-2 \eta)$ and hence $\mu \in \Delta(1, \delta)$ where $\delta^{2}+(2-2 \eta)^{2}=2^{2}$, that is, $\delta=2 \sqrt{2 \eta-\eta^{2}}$.

Let $\epsilon>0$ and choose $\delta$ according to Lemma 5.8 where $\langle z, z\rangle_{a} \leq$ $\langle e, e\rangle_{a}=n$. Choose $\eta>0$ according to the observation above so that $\frac{1}{2}|1+\mu|>1-\eta$ implies $\mu \in \Delta(1, \delta)$.

Now, suppose $\|h(z)\|>1-\eta$ so that $\frac{1}{2}\left|1+\frac{\langle z, e\rangle_{a}}{\langle e, e\rangle_{a}}\right|>1-\eta$. Then $\left|1-\frac{\langle z, e\rangle_{a}}{\langle e, e\rangle_{a}}\right|<\delta$ and thus $\|z-e\|_{a}<\epsilon$. As $\|z-e\| \leq\|z-e\|_{a}$ we conclude $\|h\|_{B \backslash B(e, \epsilon)}<1-\eta<1$ as required.

We are now in a position to prove that the set of extreme points $\Gamma$ is the infinite dimensional analogue of the Bergmann-Shilov boundary for finite rank JB*-triples.

Theorem 5.10. Let $Z$ be a finite rank $J B^{*}$-triple. Then the set $\Gamma$ of extreme points of $\bar{B}$ is the smallest closed subset $\Lambda$ of $\bar{B}$ such that

$$
\sup \{\|f(z)\|: z \in \bar{B}\}=\sup \{\|f(z)\|: z \in \Lambda\}
$$

for all $f: B \mapsto \mathbb{C}$ holomorphic on $B$ with continuous extension to $\partial B$.

Proof. Corollary 4.1 shows that $\Gamma$ is a (closed) set satisfying (10). Now let $\Lambda$ be any closed set in $\bar{B}$ satisfying (10). Suppose $e \in \Gamma$ but $e \notin \Lambda$. Then as $\Lambda$ is closed, $B(e, \delta) \cap \Lambda=\emptyset$ for some $\delta>$ 0 . By Proposition 5.9, there exists $h \in \mathcal{H}(Z, \mathbb{C})$ such that $\|h\|_{\Lambda} \leq$ $\|h\|_{\bar{B} \backslash B(e, \delta)}<\|h\|_{\bar{B}}$, which is a contradiction to (10). We conclude $e \in \Lambda$ and consequently, $\Gamma \subset \Lambda$ as required.

If a finite rank $J B^{*}$-triple $Z$ contains a unitary tripotent then $\Gamma_{1}$ is a non-empty closed subset of $\Gamma$ which, by Proposition 4.1 part (2), satisfies (10). By Theorem 5.10 then, $\Gamma_{1}$ cannot be a proper subset of $\Gamma$ and we have the following consequence.

Corollary 5.11. If a finite rank $J B^{*}$-triple has a unitary tripotent then all of its maximal tripotents are unitary. 
Corollary 5.12. Let $Z$ be a finite rank $J B^{*}$-triple with open unit ball $B$ and $v \in \partial B$. Then $\Gamma \subseteq \overline{G_{v}^{0}}$.

Proof. From Proposition 4.3, $\overline{G_{v}^{0}}$ is a closed determining set satisfying (10) so the result follows by Theorem 5.10 .

\section{REFERENCES}

[1] DinEEN, S. Complete holomorphic vector fields on the second dual of a Banach space. Math. Scand. 59 (1986), 131-142.

[2] Dineen, S. The Schwarz Lemma. Oxford University Press, 1989.

[3] Friedman, Y., And Russo, B. Structure of the predual of a $J B W^{*}$-triple. J. Reine Angew. Math. 356 (1985), 67-89.

[4] Friedman, Y., and Russo, B. The Gelfand Naimark theorem for JB"-triples. Duke Math. J. 53, 1 (1986), 139-148.

[5] Hamada, H., Honda, T., ANd Kohr, G. Linear invariance of locally biholomorphic mappings in the unit ball of a JB*-triple. J. Math. Anal. Appl. 385, 1 (2012), 326-339.

[6] HaRRIS, L. Bounded symmetric homogeneous domains in infinte dimensional spaces. Lecture Notes in Maths. 364 (1973), 13-40.

[7] Kaup, W. Über die Klassifikation der symmetrischen hermiteschen Mannigfaltigkeiten unendlicher dimension. I. Math. Ann. 257, 4 (1981), 463-486.

[8] Kaup, W. A Riemann mapping theorem for bounded symmetric domains in complex Banach spaces. Math. Z. 138 (1983), 503-529.

[9] Kaup, W. On spectral and singular values in $J B^{*}$-triples. Proc. Roy. Irish Acad. Sect. A 96, 1 (1996), 95-103.

[10] Kaup, W., AND SAUTER, J. Boundary structure of bounded symmetric domains. Manuscripta Math. 101, 3 (2000), 351-360.

[11] Kaup, W., AND UpmeIER, H. Banach spaces with biholomorphically equivalent unit balls are isomorphic. Proc. Amer. Math. Soc. 58 (1976), 129-133.

[12] Loos, O. Bounded symmetric domains and Jordan pairs. University of California at Irvine, Lecture Notes, 1977.

[13] Mackey, M. Homotopes of JB*-triples and a Russo-Dye theorem. AsianEuropean Journal of Mathematics 2, 3 (2009), 465-475.

[14] ReICH, S., AND ShoikHET, D. Nonlinear semigroups, fixed points, and geometry of domains in Banach spaces. Imperial College Press, London, 2005.

[15] Russo, B., AND Dye, H. A. A note on unitary operators in $C^{*}$-algebras. Duke Math. J. 33 (1966), 413-416.

[16] Siddiqui, A. A. Average of two extreme points in $J B W^{*}$-triples. Proc. Japan Acad. Ser. A Math. Sci. 83, 9-10 (2007), 176-178.

Email address: macǩey@maths.ucd.ie, pmellon@maths.ucd.ie 